\title{
IMPLICACIONES EDUCATIVAS DE LA TEORÍA SOCIOCULTURAL DE VIGOTSKY
}

Ana Lupita Chaves Salas

Resumen: El propósito de este artículo es analizar la teoría sociocultural del Lev Vigotsky y reflexionar sobre las implicaciones educativas de dicha teoría en los procesos de enseñanza y aprendizaje que se organizan en la escuela. Con base en los postulados de Vigotsky, se invita a las docentes y los docentes a repensar su práctica pedagógica y a promover cambios en su labor profesional con el fin de ofrecer una educación más contextualizada y significativa que ayude a formar personas críticas y creativas que contribuyan a construir una sociedad más democrática y solidaria.

\section{Introducción}

Las transformaciones a nivel mundial en el campo económico, político, social, ambiental y cultural demandan estrategias educativas distintas a las actuales, desde el nivel inicial hasta el universitario, que partan del análisis de la realidad y del estudio de teorías que contribuyan a interpretar y a enriquecer la práctica pedagógica como proyecto político-social. Al respecto Solé y Coll indican "...necesitamos teorías que nos sirvan de referente para contextualizar y priorizar metas y finalidades; planificar la actuación; para analizar su desarrollo e irlo modificando en función de lo que ocurre y para tomar decisiones acerca de la adecuación de todo ello" (1998:9). Se trata, por lo tanto, de analizar teorías que nos lleven a reflexionar sobre nuestras creencias y prácticas pedagógicas, con el propósito de ofrecer una educación más humana y contextualizada.

En tal perspectiva, el propósito de este artículo es analizar la teoría sociocultural de Lev Vigotsky que indica que el desarrollo del ser humano está íntimamente ligado con su interacción en el contexto sociohistórico-cultural, y reflexionar sobre las implicaciones educativas de dicha teoría en los procesos de enseñanza y aprendizaje que organizamos en la escuela, pues como bien lo señala Moll (1993), para Vigotsky la educación implica el desarrollo potencial del sujeto, y la expresión y el crecimiento de la cultura humana. 


\section{La teoría sociocultural de Vigotsky}

Lev Semionovich Vigotsky (1885-1934), psicólogo soviético que se interesó por estudiar las funciones psíquicas superiores del ser humano -memoria, atención voluntaria, razonamiento, solución de problemas- formuló una teoría a fines de los años veinte, en la que planteaba que el "desarrollo ontogenético de la psiquis del hombre está determinado por los procesos de apropiación de las formas histórico-sociales de la cultura; es decir Vigotsky articula los procesos psicológicos y los socioculturales y nace una propuesta metodológica de investigación genética e histórica a la vez" (Matos, 1996:2), de tal manera para este autor, las funciones superiores del pensamiento son producto de la interacción cultural. Por influencia del marxismo, Vigotsky indica que para comprender la psiquis y la conciencia se debe analizar la vida de la persona y las condiciones reales de su existencia, pues la conciencia es "un reflejo subjetivo de la realidad objetiva" y para analizarla se debe tomar como "un producto sociocultural e histórico, a partir de una concepción dialéctica del desarrollo" (Matos, 1996:3).

Vigotsky planteó el fundamento epistemológico de su teoría indicando que "el problema del conocimiento entre el sujeto y el objeto se resuelve a través de la dialéctica marxista (S-O), donde el sujeto actúa (persona) mediado por la actividad práctica social (objetal) sobre el objeto (realidad) transformándolo y transformándose a sí mismo" (Matos, 1996:4). En este proceso de conocimiento son esenciales el uso de instrumentos socioculturales, especialmente de dos tipos: las herramientas y los signos. Las herramientas producen cambios en los objetos y los signos transforman internamente al sujeto que ejecuta la acción. Los signos son instrumentos psicológicos producto de la interacción sociocultural y de la evolución, como es el lenguaje, la escritura y el cálculo, entre otros (Barquero, 1996).

Dentro de esta teoría, el ser humano al entrar en contacto con la cultura a la que pertenece se apropia de los signos que son de origen social para posteriormente internalizarlos. Vigotsky indica "el signo siempre es inicialmente un medio de vinculación social, un medio de acción sobre los otros y solo luego se convierte en un medio de acción sobre sí mismo" (1978:141).

El niño y la niña se van apropiando de las manifestaciones culturales que tienen un significado en la actividad colectiva, es así como "los procesos psicológicos superiores se desarrollan en los niños a través de la enculturación de las prácticas sociales, a través de la adquisición de la tecnología de la sociedad, de sus signos y herramientas, y a través de la educación en todas sus formas" (Moll, 1993:13).

La apropiación es sinónimo de adaptación que se da mediante procesos culturales y naturales. Leontiev menciona que "El proceso de apropiación realiza la necesidad principal y el principio fundamental del desarrollo ontogenético humano: la reproducción de las aptitudes y propiedades del individuo de las propiedades y aptitudes históricamente formadas por la especie humana, incluyendo la aptitud para comprender y utilizar el lenguaje." (1983:136 citado por Barquero, 1996:156).

Vigotsky (1978) señala que en el desarrollo psíquico del niño y la niña toda función aparece en primera instancia en el plano social y posteriormente en el psicológico, es decir se da al inicio a nivel interpsíquico entre los demás y posteriormente al interior del niño y de la niña en un plano intrapsíquico, en esta transición de afuera hacia dentro se transforma el proceso mismo, cambia su estructura y sus funciones. Este proceso de internalización, Vigotsky lo llamó "Ley genética general del desarrollo psíquico (cultural)", donde el principio social está sobre el principio natural-biológico, por lo tanto las fuentes del desarrollo psíquico de la persona no están en el sujeto mismo sino en el sistema de sus relaciones sociales, en el sistema de su comunicación con los otros, en su actividad colectiva y conjunta con ellos (Matos, 1996). Al respecto Luria (s.f., p. 123 ) afirma: 
La actividad psicológica del niño se forma bajo la influencia, por una parte de las cosas que lo rodean, cada una de las cuales representa la historia materializada de la vida espiritual de centenares de generaciones, y por otra parte, del derredor, por las relaciones que el niño tendrá con él. Al nacer el niño no es una persona autística que sólo en forma gradual entrará en la cultura; desde el principio mismo de su vida es tomado por la red de las influencias culturales, y solo en forma progresiva ha de distinguirse como criatura independiente, cuyo mundo espiritual continúa siendo socialmente modelado.

Vigotsky considera que el momento más significativo en el desarrollo del infante, es cuando el lenguaje y la actividad práctica convergen, siendo anteriormente dos líneas de desarrollo totalmente independientes, "en un momento dado se unen y el lenguaje se vuelve racional y el pensamiento verbal. El desarrollo que hasta ahora era biológico se vuelve sociohistórico ya que por medio del lenguaje racional, la sociedad inyecta en el individuo las significaciones que ha elaborado en el transcurso de su historia" (Morales, 1990:11).

De acuerdo con esta teoría, el lenguaje se da en un primer momento a nivel social, luego es egocéntrico y más adelante interiorizado (Vigotsky, 1978). Cuando el infante inicia la comunicación verbal, el lenguaje sigue a las acciones, es provocado y dominado por la actividad; en estadios superiores surge una nueva relación entre la palabra y la acción, ya que el lenguaje guía, determina y domina el curso de la acción y aparece su función planificadora, de tal manera el lenguaje es esencial para el desarrollo cognoscitivo del niño (Morales, 1990).

Vigotsky considera que el significado de la palabra es clave para estudiar las relaciones internas del discurso y el pensamiento, se basa en "la proposición de que el significado es una condición necesaria tanto para el pensamiento como para el discurso... es importante destacar que la búsqueda del sentido y significado juega un papel importante en la teorización de Vigotsky, en especial en su importancia ontogenética" (Moll, 1993:19). Para este autor es fundamental el papel del sentido y el significado en el desarrollo de la percepción en los niños y las niñas, los usos cognitivos de los signos y las herramientas, el desarrollo de la escritura y el juego, al cual le dio gran importancia para la interiorización y apropiación del ambiente durante los primeros años de vida.

\section{Implicaciones educativas}

Vigotsky (1977) fue profesor en varias instituciones de enseñanza, se interesó por los problemas de aprendizaje, de desarrollo y por los procesos educativos en niños y niñas normales y con necesidades especiales, siempre le interesó relacionar la psicología científica con la labor pedagógica, partió de la observación de la realidad educativa para hacer sus propuestas en el campo de la psicología. Para Vigotsky las escuelas (y otras instituciones educativas informales) representaban los mejores "laboratorios culturales" para estudiar el pensamiento y modificarlo mediante la acción cooperativa entre adultos e infantes.

El estudio del cambio educativo tuvo un gran significado teórico y metodológico en su enfoque ya que representó la reorganización de un sistema social clave y modos asociados de discurso, con consecuencias potenciales para el desarrollo de nuevas formas de pensamiento. Su preocupación por el cambio práctico surge por su orientación marxista y por el contexto en que este autor desarrolla su obra, el de la revolución rusa y los graves problemas que enfrentaba el país (Moll, 1992).

Para este autor, la enseñanza y la educación constituyen formas universales del desarrollo psíquico de la persona y el instrumento esencial de enculturación y humanización. Al respecto Rivière afirma:

Para él, las funciones superiores eran el resultado de la enculturación, de la influencia cultural en el aprendizaje y el desarrollo, y sólo podían ser explicadas en su génesis, por su historia, situándolas en su contexto originante. Por tanto, la bumanización era un producto de la educación formal e informal, concebida en términos de interacción... Al mismo tiempo, sentía que el objetivo pragmático esencial de la propia Psicología era la mejora y el perfeccionamiento de la educación real, que era como decir la mejora y el perfeccionamiento del hombre mismo (1988, p. 18). 
Jerome Bruner señala que "la concepción de Vygotsky del desarrollo es al mismo tiempo una teoría de la educación ...su teoría educacional es una teoría de transmisión cultural como también una teoría de desarrollo. Ya que "educación" no sólo implica para Vygotsky el desarrollo del potencial del individuo sino la expresión y el crecimiento histórico de la cultura humana de la que surge el Hombre" (citado por Moll, 1993:13).

Para Vigotsky lo que determina el desarrollo ontogenético del ser humano tiene la siguiente estructura: actividad colectiva y comunicación - cultura (signos) - apropiación de la cultura (enseñanza y educación) actividad individual - desarrollo psíquico del individuo. Esta estructura abstracta transmite una especificidad histórica al desarrollo de la psiquis de los individuos que viven en distintas épocas y en diferentes culturas (Matos, 1996).

El concepto vigotskyano que tiene mayor aplicabilidad en el campo educativo es la zona de desarrollo próximo (ZDP). Este concepto "designa las acciones del individuo que al inicio él puede realizar exitosamente sólo en interrelación con otras personas, en la comunicación con éstas y con su ayuda, pero que luego puede cumplir en forma totalmente autónoma y voluntaria" (Matos, 1996:8).

Vigotsky planteaba dos niveles de desarrollo en los infantes: el nivel actual de desarrollo y la zona de desarrollo próximo, la que se encuentra en proceso de formación, es el desarrollo potencial al que el infante puede aspirar.

Este concepto es básico para los procesos de enseñanza y aprendizaje pues la educadora y el educador deben tomar en cuenta el desarrollo del estudiante en sus dos niveles: el real y el potencial para promover niveles de avance y autorregulación mediante actividades de colaboración como lo proponía Vigotsky. Moll (1993:20) menciona tres características para crear ZDP:

1. Establecer un nivel de dificultad. Este nivel, que se supone que es el nivel próximo, debe ser algo desafiante para el estudiante, pero no demasiado difícil.
2. Proporcionar desempeño con ayuda. El adulto proporciona práctica guiada al estudiante con un claro sentido del objetivo o resultado de su desempeño. 3. Evaluar el desempeño independiente. El resultado más lógico de una zona de desarrollo próximo es que el infante se desempeñe de manera independiente.

De acuerdo con Vigotsky (1977) el aprendizaje precede al desarrollo. Entre el aprendizaje y el desarrollo existe una relación de tipo dialéctica. Una enseñanza adecuada contribuye a crear zonas de desarrollo próximo; "es decir va a servir de imán para hacer que el nivel potencial de desarrollo del educando se integre con el actual. Esta modificaciones, a su vez pueden promover progresos en el desarrollo cognoscitivo general. Como han señalado algunos, la ZDP es un diálogo entre el niño y su futuro, entre lo que es capaz de hacer hoy y lo que será capaz de hacer mañana y no entre el niño y su pasado" (Matos, 1996:11).

Vigotsky considera a la educación formal, es decir a la escuela, como fuente de crecimiento del ser humano, si en ella, se introducen contenidos contextualizados, con sentido y orientados no al nivel actual de desarrollo del párvulo, sino a la zona de desarrollo próximo. Para él lo esencial no es la transferencia de habilidades de los que saben más a los que saben menos sino es el uso colaborativo de las formas de mediación para crear, obtener y comunicar sentido (Moll, 1993). La enseñanza debe apuntar fundamentalmente no a lo que el infante ya conoce o hace ni a los comportamientos que ya domina, sino aquello que no conoce, no realiza o no domina suficientemente. Es decir, debe ser constantemente exigente con las estudiantes y los estudiantes y ponerlos ante situaciones que les obliguen a implicarse en un esfuerzo de comprensión y de actuación. Además aclara que la enseñanza y el desarrollo son el resultado directo de la actividad de la estudiante y el estudiante en su relación con el mundo circundante.

Dentro de esta teoría se percibe al infante como un ente social, activo, protagonista y 


\section{$12: 33 \mathrm{pm}$}

Página 63

CHAVES: Implicaciones educativas de la teoría sociocultural de Vigotsky

producto de múltiples interrelaciones sociales en las que ha participado a lo largo de su vida. El infante es una persona que reconstruye el conocimiento, el cual primero se da en el plano interindividual y posteriormente en el plano intraindividual para usarlo de manera autónoma. En la interacción con los otros, en diversos ámbitos sociales es que el niño y la niña aprenden y se desarrollan integralmente.

\section{El papel del educador y la educadora}

La teoría sociocultural le da énfasis a las interrelaciones sociales. En el ámbito escolar es fundamental la relación entre estudiantes y adultos. La docente y el docente son los encargados de diseñar estrategias interactivas que promuevan zonas de desarrollo próximo, para ello debe tomar en cuenta el nivel de conocimiento de las estudiantes y los estudiante, la cultura y partir de los significados que ellos poseen en relación con lo que van aprender. La docente y el docente deben provocar desafíos y retos que hagan cuestionar esos significados y sentidos y lleven a su modificación en el infante, por lo tanto es conveniente planear estrategias que impliquen un esfuerzo de comprensión y de actuación por parte de las estudiantes y los estudiantes. Esa exigencia debe ir acompañada de los apoyos y soportes de todo tipo, de los instrumentos tanto intelectuales como emocionales, que los posibiliten a superar esas exigencias, retos y desafíos (Onrubia, 1998). Para ello es importante diversificar los tipos de actividades, posibilitar la elección de tareas distintas de parte de las alumnas y los alumnos y recurrir a diversos materiales de apoyo.

La creación de ZDP se da dentro de un contexto interpersonal docente-alumno siendo el interés del facilitador trasladar al estudiante de los niveles inferiores a los superiores de la zona. Lo esencial es dar apoyo estratégico a los infantes para que logren solucionar un problema; este apoyo se puede inducir mediante el planteamiento de preguntas claves o llevando al estudiante al autocuestionamiento. Matos afirma que la participación del docente en "el proceso instruccional para la enseñanza de algún contenido (conocimiento, habilidades, procesos) en un inicio debe ser un poco directivo mediante la creación de un sistema de apoyo que J. Bruner ha denominado "andamiaje" por donde transitan los alumnos ...y posteriormente con los avances del alumno en la adquisición o interiorización del contenido, se va reduciendo su participación al nivel de simple espectador empático" (1996:10). En este contexto la enseñanza, debe entedenderse como una ayuda al proceso de aprendizaje pero sólo ayuda, ya que la enseñanza no puede sustituir la actividad mental constructiva del alumno ni ocupar su lugar (Onrubia, 1998).

De este modo la educadora y el educador asumen un papel de mediadores, de guías para que los niños y las niñas aprendan activamente en contextos sociales significativos y reales. En este proceso el lenguaje es clave como "instrumento fundamental a través del cual los participantes pueden contrastar y modificar sus esquemas de conocimiento y sus representaciones sobre aquello que se está enseñando y aprendiendo" (Onrubia, 1998:116).

\section{Reflexión final}

Los aportes teóricos de Lev Vigotsky son propuestas pertinentes para repensar la educación y la práctica pedagógica. Estos postulados coinciden en la importancia de respetar al ser humano en su diversidad cultural y de ofrecer actividades significativas para promover el desarrollo individual y colectivo con el propósito de formar personas críticas y creativas que propicien las transformaciones que requiere nuestra sociedad. Para ello es importante que en la organización de los procesos de enseñanza y aprendizaje, las docentes y los docentes tengamos en cuenta que es importante:

- Reflexionar sobre nuestras creencias y nuestra práctica pedagógica, estudiar diferentes teoría educativas con el propósito de construir o crear nuevas situaciones y diferentes formas de acción. 
- Partir de los contextos socioculturales de nuestros estudiantes para ofrecerles una educación con sentido y significado, por lo que es necesario analizar a profundidad los significados de cada cultura, tener en cuenta que en toda cultura hay elementos residuales (formaciones culturales del pasado), dominantes (los de los sectores hegemónicos que articulan todo el resto) y emergentes (innovadores) (Carusso y Dussell, 1996).

- Pensar la cultura, y sobre todo la cultura escolar cotidiana, como culturas plurales producto de la mezcla de muchos elementos heterogéneos, donde se enlaza lo objetivo y lo subjetivo, lo que llevaría a replantear las interacciones en el salón de clase, el papel de estudiantes, educadoras y educadores, la pertinencia de los contenidos y la contribución de los padres y madres de familia, de otros profesionales y de las instituciones de la comunidad.

- Ofrecer a las estudiantes y los estudiantes experiencias de aprendizaje que partan del contexto sociocultural, de su nivel de desarrollo y de lo que tiene significativo. Es importante tener presente que para que se produzca el aprendizaje es necesario provocar retos y desafíos a los educandos, que los hagan cuestionar los significados que poseen, para que los modifiquen y se desarrollen plenamente.

- Resaltar el papel del lenguaje en la construcción del conocimiento y en la acción emancipadora del ser humano, ya que el lenguaje es una manifestación cultural que transmite un significado que responde a determinados intereses, por lo tanto es necesario estar consciente del papel que juega en la construcción del conocimiento y de la subjetividad. Es necesario tener presente que el lenguaje sirve para organizar, construir y transformar el pensamiento, para aprender, comunicar y compartir experiencias con los demás. Desde esta perspectiva, el lenguaje cobra un papel protagónico como herramienta para crear las condiciones propicias para el aprendizaje y el desarrollo.

- Crear ambientes de aprendizaje, que provoquen la actividad mental y física de las alumnas y los alumnos, el diálogo, la reflexión, la crítica, la cooperación y participación, la toma de consciencia y la autorregulación; ambientes que contribuyan a clarificar, elaborar, reorganizar y reconceptualizar significados que permitan interpretar el mundo.

- Concebir al educando como una ser activo, protagonista, reflexivo producto de variadas interrelaciones sociales que ocurren en un contexto histórico-cultural específico y que reconstruye el conocimiento con las otras y los otros.

En síntesis, se trata de pensar y repensar nuestra práctica pedagógica con el fin de ofrecer una educación más humana, que respete la diversidad cultural en todas sus dimensiones, que resalte el papel del lenguaje en la construcción del significado y el conocimiento, que promueva el diálogo, la crítica, la participación y que ayude a formar personas críticas y creativas que contribuyan a construir una sociedad más democrática comprometida con el desarrollo humano y natural de nuestro mundo.

\section{Referencias bibliográficas}

Barquero, R. Vigotsky y el aprendizaje escolar Buenos Aires, Argentina: Aique. 1996.

Caruso M. y Dussel I. De Sarmiento a los Simpsons. Cinco conceptos para pensar la educación contemporánea. Buenos Aires, Argentina: Kapelusz. 1996.

Castorina, J. y otros. Piaget-Vigotsky: contribuciones para plantear el debate. Buenos Aires: Paidós. 1996. 
Coll, C. Y Solé, Isabel. "Los profesores y la concepción constructivista". En $E l$ constructivismo en el aula. $8^{a}$ edición. Barcelona, España: GRAÓ. 1998.

Luria, A.R. Lenguaje y pensamiento. Segunda Edición. Barcelona, España: Martínez Roca, S. A. 1985.

Luria, A. (s.f) "Vygosky y las funciones psíquicas superiores". San José, Costa Rica: Proyecto SIMED (mimeo).

Matos, J. El paradigma sociocultural de L.S. Vigostky y su aplicación en la educación (mimeo). Heredia, Costa Rica: Universidad Nacional. 1995.

Moll, L. Vygotsky y la educación (2 E.) Buenos Aires: Aique. 1993.

Morales, P. El papel del lenguaje en el desarrollo cognoscitivo: anteposición de la perspectiva piagetiana frente a la perspectiva soviétiva (mimeo). Puerto Rico: Recinto de Río Piedras. Universidad de Puerto Rico. 1990.

Onrubia, Javier. "Enseñar: crear zonas de desarrollo próximo e intervenir en ellas". En El constructivismo en el aula. $8^{a}$ edición. Barcelona, España: GRAÓ. 1998.

Rivière, A. La psicología de Vygotski. (3 ${ }^{\mathrm{a}}$ E.) Madrid: Visor. 1988.

Rodríguez, Wanda. "La perspectiva constructivista en la educación" Pedagogía. Vol. 32. San Juan Puerto Rico: Recinto de Río Piedras, Facultad de Educación. Universidad de Puerto Rico. 1998.

Vigotsky, L.S. Pensamiento y Lenguaje. Buenos Aires, Argentina: La Pleyade. 1978. 